ANNALES

POLONICI MATHEMATICI

$96.3(2009)$

\title{
On the extendability of quadratic polynomial mappings of the plane
}

\author{
by EwA LigockA (Warszawa)
}

\begin{abstract}
We shall prove, using the result from our previous paper [Ann. Polon. Math. 88 (2006)], that for a quadratic polynomial mapping $Q$ of $\mathbb{R}^{2}$ only the geometric shape of the critical set of $Q$ determines whether the complexification of $Q$ can be extended to an endomorphism of $\mathbb{C P}^{2}$. At the end of the paper we describe some interesting classes of quadratic polynomial mappings of $\mathbb{R}^{2}$ and give some examples.
\end{abstract}

1. Introduction. A quadratic polynomial mapping of the plane $\mathbb{R}^{2}$ into itself is a map

$$
Q(x, y)=\left(q_{1}(x, y), q_{2}(x, y)\right)
$$

where $q_{1}$ and $q_{2}$ are polynomials and

$$
\max \left(\operatorname{deg} q_{1}, \operatorname{deg} q_{2}\right)=2 .
$$

Sometimes it is convenient to identify $\mathbb{R}^{2}$ with the complex plane $\mathbb{C}$, put $z=x+i y$ and write $Q$ in the form $Q(z)=a z^{2}+b|z|^{2}+c \bar{z}+d z+e \bar{z}+f=$ $Q_{2}(z)+Q_{1}(z)+Q_{0}$ where $Q_{2}(z)=a z^{2}+b|z|^{2}+c \bar{z}^{2}, Q_{1}(z)=d z+e \bar{z}, Q_{0}=f$.

Following [Li2] and [Li3] we shall call the mapping extendable if its complexification $f(z, w)$ extends to a polynomial endomorphism of the complex projective space $\mathbb{C P}^{2}$. We shall say that $Q$ is properly extendable if $f(z, w)$ acts on $\mathbb{C P}^{2}$ as a finite Blaschke product.

We shall consider the critical set of $Q$, equal to the zero set of the Jacobi determinant $J Q$. We have

$$
J Q=\operatorname{det}\left[\begin{array}{cc}
\frac{\partial q_{1}}{\partial x} & \frac{\partial q_{1}}{\partial y} \\
\frac{\partial q_{2}}{\partial x} & \frac{\partial q_{2}}{\partial y}
\end{array}\right]=\left|\frac{\partial Q}{\partial z}\right|^{2}-\left|\frac{\partial Q}{\partial \bar{z}}\right|^{2} .
$$

Theorem 2.1 of [Li2] yields

2000 Mathematics Subject Classification: Primary 30C99, 30D50, 30C10, 32H02; Secondary 32H50, 32D15, 30C62.

Key words and phrases: quadratic polynomial mapping, complexification, quasiregular mapping, harmonic polynomial, invariant triangle, simplex mapping. 
THEOREM 0. A quadratic polynomial mapping $Q$ is extendable iff one of the following conditions holds:

(1) $Q_{2}(z)=\alpha(z-p \bar{z})(z-q \bar{z})$ where $\alpha \neq 0,|p|^{2} \neq 1,|q|^{2} \neq 1, p \bar{q} \neq 1$,

(2) $Q_{2}(z)=\alpha \bar{z}(z-p \bar{z}), \alpha \neq 0,|p| \neq 0,1$,

(3) $Q_{2}(z)=\alpha \bar{z}^{2}, \alpha \neq 0$.

From the results of [Li3] we also have

Proposition 0. A quadratic polynomial mapping $Q$ is properly extendable iff

$$
Q_{2}(z)=\alpha(z-p \bar{z})(z-q \bar{z}), \quad \alpha \neq 0,|p|<1,|q|<1 .
$$

The aim of the present note is to show that for quadratic polynomial map $Q$ the extendability of $Q$ depends only on the geometric shape of the critical set of $Q$.

We shall also describe some important classes of such mappings and give some examples.

2. The critical set and affine conjugations. If $Q$ is a quadratic polynomial map then $J Q$ is a polynomial of degree 2,1 or 0 . Hence the zero set of $J Q$ can be equal to

(1) one point,

(2) an ellipse,

(3) a hyperbola,

(4) a pair of intersecting lines,

(5) a parabola,

(6) a pair of parallel lines,

(7) a single line,

(8) an empty set,

(9) the whole plane.

We name the zero set of $J Q$ the critical set of $Q$.

We shall prove that if the critical set of $Q$ is a single point then either $Q$ or $\bar{Q}$ is quasiregular ( $Q$ is quasiregular iff $\left|\frac{\partial Q}{\partial \bar{z}} / \frac{\partial Q}{\partial z}\right|<\delta<1$ a.e. on $\left.\mathbb{C}\right)$. The paper [Li1] was devoted to studying such quadratic mappings.

When dealing with cases (2)-(7) we shall use the following algebraic fact:

(*) Let $k$ be any field, $f \in k[x, y]$ a prime polynomial, $g \in k[x, y]$ any polynomial. If $f$ does not divide $g$ then the equations $f(x, y)=0$ and $g(x, y)=0$ can have only a finite number of common solutions (see [Sha, Ch. 1, p. 1]).

Suppose now that we are in case (2) and let $C$ denote the critical ellipse of $Q$. There exists a nondegenerate affine map $h: \mathbb{R}^{2} \rightarrow \mathbb{R}^{2}$ which maps the unit circle onto $C$. Let us consider the map $Q^{0}=h^{-1} \circ Q \circ h$. 
The critical set of $Q^{0}$ is equal to the unit circle $T=\left\{z \in \mathbb{C}:|z|^{2}-1=0\right\}$. The algebraic fact $(*)$ implies that

$$
J Q^{0}(z)=\gamma\left(|z|^{2}-1\right), \quad \gamma \neq 0, \gamma \in \mathbb{R} .
$$

The polynomial $Q^{0}$ can be written as

$$
Q^{0}(z)=a z^{2}+b|z|^{2}+c \bar{z}^{2}+d z+e \bar{z}+f .
$$

Simple calculations show that $b=0,|a| \neq|c|,|e|=2|a|,|\alpha|=2|c|$ and $a \bar{d}=\bar{c} e$. Hence $Q^{0}$ is a harmonic polynomial

The above calculations are based on the following formula:

$(* *)$ If $P(z)=a z^{2}+b|z|^{2}+c \bar{z}^{2}+d z+e \bar{z}+f$ then

$$
\begin{aligned}
J P(z)= & z^{2}(2 a \bar{b}-2 \bar{c} b)+|z|^{2}\left(4|a|^{2}-4|c|^{2}\right) \\
& +\bar{z}^{2}(2 \bar{a} b-2 c \bar{b})+z(2 a \bar{d}+d \bar{b}-b \bar{e}-2 \bar{c} e) \\
& +\bar{z}(2 \bar{a} d+\bar{d} b-\bar{b} e-2 c \bar{e})+|d|^{2}-|e|^{2} .
\end{aligned}
$$

In case (3), $Q$ is affinely conjugate to $Q^{0}$ with

$$
J Q^{0}(z)=\gamma\left(z^{2}+\bar{z}^{2}-1\right), \quad \gamma \in \mathbb{R}, \gamma \neq 0 .
$$

In case (4), $Q$ is affinely conjugate to $Q^{0}$ with

$$
J Q^{0}(z)=\gamma\left(z^{2}+\bar{z}^{2}\right), \quad \gamma \in \mathbb{R}, \gamma \neq 0 .
$$

In both cases we have, by $(* *)$,

$$
|a|=|c| \quad \text { and } \quad 2 a \bar{b}-2 \bar{c} b=\gamma .
$$

In case (6), $Q$ is affinely conjugate to $Q^{0}$ for which

$$
J Q^{0}(z)=\gamma\left((z+\bar{z})^{2}+z+\bar{z}\right), \quad \gamma \in \mathbb{R}, \gamma \neq 0 .
$$

In case (5), $Q$ is affinely conjugate to $Q^{0}$ with

$$
J Q^{0}(z)=\gamma\left((z+\bar{z})^{2}-\frac{z-\bar{z}}{2 i}\right), \quad \gamma \in \mathbb{R}, \gamma \neq 0 .
$$

In case (7), there are two possibilities:

(7i) $Q$ is affinely conjugate to $Q^{0}$ with

$$
J Q^{0}(z)=\gamma_{1} x^{2}=\gamma_{1}\left(\frac{z+\bar{z}}{2}\right)^{2},
$$

(7ii) $Q$ is affinely conjugate to $Q^{0}$ with

$$
J Q^{0}(z)=\gamma x=\gamma\left(\frac{z+\bar{z}}{2}\right) .
$$

All the above statements can be proved in the same way as in case (2). 
3. The extendability. The aim of the present paper to prove the following

TheOrem 1. A quadratic polynomial mapping $Q$ is extendable iff its critical set is equal to one of the following sets:

(1) a single point,

(2) an ellipse,

(3) a hyperbola,

(4) a pair of intersecting lines.

THEOREM 2. If the critical set of a quadratic polynomial mapping $Q$ is bounded (i.e. it is either a point or an ellipse) then either $Q$ or $\bar{Q}$ is properly extendable.

In order to prove Theorem 1 we shall show

Proposition 1. If the critical set of a quadratic polynomial map $Q$ is a single point then either $Q$ or $\bar{Q}$ is quasiregular.

Proposition 1 can be formulated in a slightly different way:

Proposition 1'. A quadratic polynomial mapping $Q$ is quasiregular iff the critical set of $Q$ consists of a single point and there exists $z_{0} \in \mathbb{C}$ such that $J Q\left(z_{0}\right)>0$.

REMARK 1. Theorem 1 is not valid for polynomial mappings of degree greater than two. If, for example, $Q(z)=z|z|^{2}$ then the critical set of $Q$ is $\{0\}$ but $Q$ is not extendable.

REMARK 2. A $C^{1}$-version of the argument principle shows that if $Q$ or $\bar{Q}$ is properly extendable then $Q$ maps $\mathbb{C}$ onto $\mathbb{C}$. This is not true for $Q$ extendable but not properly extendable. For example, the mapping $Q(z)=$ $z(2 \bar{z}+z)=z^{2}+2|z|^{2}$ is extendable by Theorem 0 , but does not have values in the left half-plane $\{z: \operatorname{Re} z<0\}$. Note that if the critical set of $Q$ is a hyperbola or a pair of intersecting lines then $Q$ is extendable but not properly extendable ( $\bar{Q}$ is not properly extendable either).

Remark 3. It is obvious that $Q$ is extendable iff $\bar{Q}$ is extendable. However, if one wants to study the dynamics of $Q$ and $\bar{Q}$ under iteration then one observes the great difference betwen them. It suffices to compare the Mandelbar set for the family $\left\{\bar{z}^{2}+c\right\}_{c \in \mathbb{C}}$ (see [CHRS] and [N]) with the classical Mandelbrot set (further information on the dynamics of antiholomorphic mappings can be found in $[\mathrm{NS}])$.

\section{Proofs}

Proof of Proposition 1. By conjugating $Q$ via a shift we can assume that the critical set is $\{0\}$. By taking, if needed, $\bar{Q}$ instead of $Q$ we can assume 
that $J Q>0$ on $\mathbb{C} \backslash\{0\}$. We have $J Q=J Q_{2}+l(z)$, where $l(z)$ is a linear map from $\mathbb{C}$ into $\mathbb{R}$.

If $l(z) \not \equiv 0$ then there exists $a \in \mathbb{C} \backslash\{0\}$ for which $l(a)=-\delta<0$ and $J Q_{2}(a)=\varepsilon>0$. Since $J Q_{2}$ is a quadratic form, we have

$$
J Q(t a)=t^{2} J Q_{2}(a)+t l(a)=t^{2} \varepsilon-t \delta<0
$$

for $0<t<\delta / \varepsilon$. This contradiction implies that $l(z) \equiv 0$ and $J Q=J Q_{2}$. There exists $\gamma>0$ such that

$$
J Q=\left|\frac{\partial Q}{\partial z}\right|^{2}-\left|\frac{\partial Q}{\partial \bar{z}}\right|^{2}>\gamma>0 \quad \text { on } T=\{z:|z|=1\} .
$$

Hence

$$
\frac{\left|\frac{\partial Q}{\partial \bar{z}}\right|^{2}}{\left|\frac{\partial Q}{\partial z}\right|^{2}}<\frac{\left|\frac{\partial Q}{\partial \bar{z}}\right|^{2}}{\gamma+\left|\frac{\partial Q}{\partial \bar{z}}\right|^{2}}<k<1 \quad \text { on } T \text {. }
$$

Since $J Q=J Q_{2}$ the same inequality holds for $Q_{2}$; since $Q_{2}$ is homogeneous, it is valid on $\mathbb{C}$ and $Q_{2}$ is quasiregular. Let $Q=a z^{2}+b|z|^{2}+c \bar{z}^{2}+d z+e \bar{z}+f$. By formula $(* *),|\alpha|^{2}-|e|^{2}=0$ and

$$
l(z)=\operatorname{Re}(2 a \bar{d}+d \bar{b}-b \bar{e}-2 \bar{c} e) z \equiv 0,
$$

which implies that $2 a \bar{d}+d \bar{b}-b \bar{e}-2 \bar{c} e=0$. We have already proved that $Q_{2}(z)=a z^{2}+b|z|^{2}+c \bar{z}^{2}$ is quasiregular. This implies that $Q_{2}^{*}(z)=a z^{2}-$ $b|z|^{2}+c \bar{z}^{2}$ is also quasiregular (see [Li1]).

We have $2 a \bar{d}-b \bar{e}=2 \bar{c} e-d \bar{b}$ and if $d \neq 0$ and $e \neq 0$ then

$$
\bar{d}\left(2 a-b \frac{\bar{e}}{\bar{d}}\right)=\left(2 \bar{c} \frac{e}{d}-\bar{b}\right) d \quad \text { since }|d|=|e| .
$$

We can put $\bar{e} / \bar{d}=e^{i \theta}$ to obtain

$$
\left|2 a-b e^{i \theta}\right|=\left|2 c \cdot e^{i \theta}-b\right| .
$$

However, $Q_{2}^{*}(z)$ is quasiregular and

$$
\left|\frac{\frac{\partial Q_{2}^{*}}{\partial \bar{z}}}{\frac{\partial Q_{2}^{*}}{\partial z}}\right|=\left|\frac{2 c \bar{z}-b z}{2 a z-b \bar{z}}\right|=\left|\frac{2 c \frac{\bar{z}}{z}-b}{2 a-b \frac{\bar{z}}{z}}\right|<m<1 .
$$

Thus we have reached a contradiction. Hence either $|d|=|e|=0$ or $2 a-$ $b e^{i \theta}=2 c e^{i \theta}-b=0$. The second possibility leads to $Q_{2}^{*}(z)=a\left(z-e^{-i \theta} \bar{z}\right)^{2}$, which is not quasiregular. This implies that $|e|=|d|=0$ and $Q(z)=$ $Q_{2}(z)+f$ is regular.

Proof of Theorem 1. (i) Proposition 1 shows that if the critical set of $Q$ is a single point then $Q$ or $\bar{Q}$ is properly extendable since by results of [Li1] each quasiregular quadratic polynomial map is properly extendable. 
(ii) We have already proved that if a critical set of $Q$ is an ellipse then $Q$ is affinely conjugate to $Q^{0}$ for which

$$
b=0, \quad|a| \neq|c|, \quad|e|=2|a|, \quad|f|=2|c|
$$

and $a \bar{d}=c e$. Hence $Q^{0}$ or $\overline{Q^{0}}$ can be written as $Q^{*}(z)=\alpha(z-p \bar{z})(z+p \bar{z})+$ $d z+e \bar{z}+f$. In the first case we have

$$
\alpha=a, c=\alpha p^{2}, \quad \text { which implies }|p|^{2} \neq 1 ;
$$

in the second case

$$
\bar{\alpha}=c, \overline{\alpha p}^{2}=a, \quad \text { and } \quad|p|^{2} \neq 1 .
$$

Thus by Theorem $0, Q^{0}$ is extendable. The complexification of an affine nondegenerate map of $\mathbb{R}^{2}$ is a nondegenerate affine map of $\mathbb{C}^{2}$ which extends to an automorphism of $\mathbb{C P}^{2}$ (which acts on $\mathbb{C P}^{2} \backslash \mathbb{C}^{2}$ as a fractional-linear map).

This implies that the conjugate map $Q$ is also extendable.

(iii) Assume now that the critical set of $Q$ is a hyperbola or a pair of intersecting lines.

In this case $Q$ is affinely conjugate to $Q^{0}$ for which $|a|=|c|$ and $2 a \bar{b}-$ $2 \bar{c} b=\gamma, \gamma \in \mathbb{R}, \gamma \neq 0$. By taking $\overline{Q^{0}}$ instead of $Q^{0}$ if necessary, we can write $Q^{0}=\alpha(z-p \bar{z})(z-q \bar{z})+$ lower order terms. We have $a=\alpha, b=-\alpha(p+q)$ and $c=\alpha p q$. Hence

$$
\begin{aligned}
& |\alpha|=|c| \Rightarrow|p q|=1,2 a \bar{b}-2 \bar{c} b=\gamma \\
& \Rightarrow|\alpha|^{2}(\overline{p q}(p+q)-\bar{p}-\bar{q})=\gamma \Rightarrow \bar{p}\left(|q|^{2}-1\right)+\bar{q}\left(|p|^{2}-1\right)=\frac{\gamma}{|\alpha|^{2}} .
\end{aligned}
$$

If $|p|=1$ or $|q|=1$ or $p \bar{q}=1$ then the left side of the last equality is zero because $|p q|=1$. This contradicts the fact that $\gamma \neq 0$ and $\alpha \neq 0$. Hence by Theorem $0, Q^{0}$ is extendable and hence so is $Q$, being affinely conjugate to $Q^{0}$. Note that we have either $|q|<1$ and $|p|>1$, or $|p|<1$ and $|q|>1$. Hence neither $Q$ nor $\bar{Q}$ can be properly extendable.

(iv) Let us assume now that the critical set of $Q$ is a parabola or a pair of parallel lines, or that the critical set of $Q$ is a single line and $Q$ is affinely conjugate to $Q^{0}$ with $J Q^{0}=\gamma_{1} x^{2}=\gamma_{1}\left(\frac{z+\bar{z}}{2}\right)^{2}$ (case (7i)). In each of these cases, $Q$ affinely conjugate to $Q^{0}$ with $J Q_{2}^{0}=\gamma(z+\bar{z})^{2}, \gamma \in \mathbb{R}, \gamma \neq 0$. As before, we can assume that

$$
Q_{2}^{0}=\alpha(z-p \bar{z})(z-q \bar{z})=a z^{2}+b|z|^{2}+c \bar{z}^{2} .
$$

We have $2 a \bar{b}-2 \bar{c} b=\gamma$ and $4\left(|a|^{2}-|c|^{2}\right)=2 \gamma$. This implies that

$$
\bar{p}\left(|q|^{2}-1\right)+\bar{q}\left(|p|^{2}-1\right)=1-|p q|^{2} \neq 0 .
$$


That means

$$
\begin{aligned}
-\operatorname{Im} p\left(|q|^{2}-1\right)-\operatorname{Im} q\left(|p|^{2}-1\right) & =0, \\
\operatorname{Re} p\left(|q|^{2}-1\right)+\operatorname{Re} q\left(|p|^{2}-1\right) & =1-|p|^{2}|q|^{2} .
\end{aligned}
$$

Let us assume that $|p| \neq 1$ and $|q| \neq 1$. Then

$$
\begin{aligned}
\operatorname{Im} p & =\frac{-\operatorname{Im} q\left(|p|^{2}-1\right)}{|q|^{2}-1}, \\
\operatorname{Re} p & =\frac{1-|p|^{2}|q|^{2}-\operatorname{Re} q\left(|p|^{2}-1\right)}{|q|^{2}-1}, \\
|p|^{2} & =\frac{(\operatorname{Im} q)^{2}\left(|p|^{2}-1\right)^{2}+\left(1-|p|^{2}|q|^{2}-\operatorname{Re} q\left(|p|^{2}-1\right)\right)^{2}}{\left(|q|^{2}-1\right)^{2}} .
\end{aligned}
$$

Put $s=|p|^{2}$. We have

$$
\begin{aligned}
s\left(|q|^{2}-1\right)^{2}= & (\operatorname{Im} q)^{2}(s-1)^{2}+\left(1-s|q|^{2}-\operatorname{Re} q(s-1)\right)^{2} \\
= & (\operatorname{Im} q)^{2}(s-1)^{2}+1+s^{2}|q|^{4}+(\operatorname{Re} q)^{2}(s-1)^{2}+2 s(s-1) \operatorname{Re} q \\
& -2 s|q|^{2}-2(s-1) \operatorname{Re} q .
\end{aligned}
$$

Hence

$$
\begin{aligned}
(s-1)\left(1-s|q|^{4}\right) & =(s-1)\left(|q|^{2}(s-1)+2 s|q|^{2} \operatorname{Re} q-2 \operatorname{Re} q\right), \\
1-s|q|^{4} & =(s-1)|q|^{2}+2 s|q|^{2} \operatorname{Re} q-2 \operatorname{Re} q, \\
2 \operatorname{Re} q+1+|q|^{2} & =s|q|^{2}\left(2 \operatorname{Re} q+1+|q|^{2}\right) .
\end{aligned}
$$

This implies that $s|q|^{2}=1$ and $|p|^{2}|q|^{2}=1$. This contradiction shows that either $|q|=1$ or $|p|=1$, and by Theorem $0, Q^{0}$ and $Q$ are not extendable.

(v) Suppose now that $J Q$ is a linear map from $\mathbb{C}$ into $\mathbb{R}$ or vanishes identically on $\mathbb{C}$ (cases (7ii) and (9)). Then $J Q_{2}$ vanishes on the whole plane and $|a|^{2}-|c|^{2}=0$ and $2 a \bar{b}-2 \bar{c} b=0$. Hence, as before,

$$
\bar{p}\left(|q|^{2}-1\right)+\bar{q}\left(|p|^{2}-1\right)=0 \text { and }|p q|=1 .
$$

If $|p| \neq 1$ then $\bar{p}\left(1 /|p|^{2}-1\right)+\bar{q}\left(|p|^{2}-1\right)=0$, so $1 / p=\bar{q}$ and $p \bar{q}=1$. Hence $Q$ is not extendable by Theorem 0 .

(vi) There remains the case when the critical set of $Q$ is empty. Then $Q$ must be a polynomial automorphism, since it has degree two. A proof of this fact can be found in [She, Chapter 3, 3.1.6]. By Proposition 2.7 of [Li2], $Q$ cannot be extendable.

Our Theorem 1 is proved.

Proof of Theorem 2. If the critical set of $Q$ is bounded then it must be a single point or an ellipse. We have already proved in the proof of Theorem 1 that in this case either $Q$ or $\bar{Q}$ is properly extendable. Conversely, if $Q$ or $\bar{Q}$ is properly extendable then $Q_{2}$ or $\bar{Q}_{2}$ is quasiregular. By the results 
of [Li1] this implies that $J Q_{2} \neq 0$ in the neighborhood of infinity. As a result, $J Q \neq 0$ in the neighborhood of infinity and therefore the critical set of $Q$ is bounded.

\section{Some classes of quadratic polynomial mappings}

(i) Quasiregular mappings. Quasiregular quadratic polynomial mappings were described in [Li1]. We only recall here that each such map is conjugate via an affine holomorphic map to a mapping $Q(z)=(z-p \bar{z})(z-q \bar{z})+c$, $|p|<1,|q|<1, c \in \mathbb{C}$. Each such $Q$ is properly extendable and its critical set is $\{0\}$. A special subclass of quasiregular quadratic polynomial maps is formed by the well-known holomorphic quadratic maps $\left\{z^{2}+c\right\}_{c \in \mathbb{C}}$ (see e.g. [CG, Chapter 8]).

(ii) Harmonic mappings. The general form of a quadratic polynomial harmonic map is $Q(z)=a z^{2}+c \bar{z}^{2}+d z+e \bar{z}+f$, and we have $J Q(z)=$ $\left(|a|^{2}-|c|^{2}\right)|z|^{2}+l(z)$ where $l(z)$ is an affine map from $\mathbb{C}$ into $\mathbb{R}$. By conjugating $Q$ via a shift we can assume that $l(z)$ is linear.

This simple fact yields immediately:

Proposition 2. Let $Q$ be a quadratic polynomial and a harmonic mapping.

(a) $Q$ is extendable iff $|a| \neq|c|$.

(b) If $Q$ is extendable then either $Q$ or $\bar{Q}$ is properly extendable and its critical set is either a single point or a circle.

(c) If $Q$ is not extendable then the critical set of $Q$ is a single line or the whole plane.

The class of harmonic quadratic polynomial maps is important because, as already proved, every quadratic polynomial map whose critical set is an ellipse is affinely conjugate to a harmonic map.

An interesting class of mappings is the class of quasiregular quadratic harmonic maps. Each such map is conjugate to $Q(z)=(z-p \bar{z})(z+p \bar{z})+c$, $|p|<1, c \in \mathbb{C}$. There is also the class of antiholomorphic quadratic maps $\left\{\bar{z}^{2}+c\right\}$ studied in [CHRS], [N], [NS]. We also mention the class $\left\{z^{2}-c \bar{z}\right\}_{c \in \mathbb{C}}$ considered by Uchimura [U].

(iii) Mappings with invariant triangles. We shall say that a quadratic polynomial mapping $Q$ has an invariant closed triangle $\triangle$ if $Q(\triangle) \subset \triangle$. We shall assume that the triangle $\triangle$ is nondegenerate, that is, has nonempty interior.

This class of mappings is interesting because such mappings are conjugate on $\triangle$ to simplex mappings given by quadratic homogeneous mappings of $\mathbb{R}^{3}$ which map the simplex $\triangle_{2}=\left\{\left(x_{1}, x_{2}, x_{3}\right) \in \mathbb{R}^{3}: x_{1} \geq 0, x_{2} \geq 0\right.$, $\left.x_{3} \geq 0, x_{1}+x_{2}+x_{3}=1\right\}$ into itself. 
Simplex mappings play an important role in the theory of population genetics (see [Ly, Chapter 8]). (Simplex mappings can be considered on $\triangle_{n-1}$ in $\mathbb{R}^{n}$ for any $n$, but we are now interested in the two-dimensional case.)

Let $F\left(x_{1}, x_{2}, x_{3}\right)=\left(F_{1}\left(x_{1}, x_{2}, x_{3}\right), F_{2}\left(x_{1}, x_{2}, x_{3}\right), F_{3}\left(x_{1}, x_{2}, x_{3}\right)\right)$ be such that $F\left(\triangle_{2}\right) \subset \triangle_{2}$ and each $F_{j}, j=1,2,3$, is a quadratic homogeneous form on $\mathbb{R}^{3}$ with values in $\mathbb{R}$. Denote by $\pi: \mathbb{R}^{3} \rightarrow \mathbb{R}^{2}$ the projection $\pi\left(x_{1}, x_{2}, x_{3}\right)=$ $\left(x_{1}, x_{2}\right)$. We have $\pi\left(\triangle_{2}\right)=\triangle_{0}=\left\{\left(x_{1}, x_{2}\right): x_{1} \geq 0, x_{2} \geq 0, x_{1}+x_{2} \leq 1\right\}$. Define now

$$
Q\left(x_{1}, x_{2}\right)=\left(F_{1}\left(x_{1}, x_{2}, 1-x_{1}-x_{2}\right), F_{2}\left(x_{1}, x_{2}, 1-x_{1}-x_{2}\right)\right) .
$$

We obtain a quadratic polynomial mapping $Q$ such that $Q\left(\triangle_{0}\right) \subset \triangle_{0}$.

If we have a quadratic polynomial mapping $Q$ for which $Q\left(\triangle_{0}\right) \subset \triangle_{0}$, we first take the mapping

$$
\bar{Q}(x, y)=\left(Q_{1}\left(x_{1}, x_{2}\right), Q_{2}\left(x_{1}, x_{2}\right), 1-Q_{1}\left(x_{1}, x_{2}\right)-Q_{2}\left(x_{1}, x_{2}\right)\right)
$$

and multiply each term of degree 0 in each $Q_{i}, i=1,2,3$, by $\left(x_{1}+x_{2}+x_{3}\right)^{2}$ and each term of degree 1 in each $Q_{i}, i=1,2,3$, by $x_{1}+x_{2}+x_{3}$. This gives homogeneous forms $F_{i}\left(x_{1}, x_{2}, x_{3}\right), i=1,2,3$. This procedure gives us a conjugation between $Q$ acting on $\triangle_{0}$ and $F$ acting on $\triangle_{2}$. (This conjugacy extends to a conjugacy between $Q$ on $\mathbb{R}^{2}$ and $F$ on the affine hyperplane $\left\{\left(x_{1}, x_{2}, x_{3}\right): x_{1}+x_{2}+x_{3}=1\right\} \subset \mathbb{R}^{3}$.)

Let $Q$ be a quadratic polynomial map with an invariant triangle $\triangle$. Let $h$ be a nondegenerate affine map $\mathbb{R}^{2}$ onto $\mathbb{R}^{2}$ such that $h(\triangle)=\triangle_{0}$. Let $Q_{1}=h^{-1} \circ Q \circ h$. Then $Q_{1}\left(\triangle_{0}\right) \subset \triangle_{0}$ and $Q_{1}$ is conjugate to some simplex map $F$ (on $\triangle$ ). Hence $Q$ is also conjugate to $F$ (on $\triangle$ ).

We have thus proved the following

Proposition 3. A quadratic polynomial mapping $Q$ with an invariant triangle $\triangle$ is conjugate on $\triangle$ to some simplex map $F$ on $\triangle_{2}$.

Among the quadratic polynomial maps with an invariant triangle there are both extendable and nonextendable maps.

ExAmple 1 (the Ulam map, see [MSU] and also [Ly, Chapter 8, Ex. 8.3.1]). Let

$$
F\left(x_{1}, x_{2}, x_{3}\right)=\left(x_{1}^{2}+2 x_{1} x_{2}, x_{2}^{2}+x_{2} x_{3}, x_{3}^{2}+2 x_{3}, x_{1}\right)
$$

and

$$
Q\left(x_{1}, x_{2}\right)=\left(x_{1}^{2}+2 x_{1} x_{2}, x_{2}^{2}+2 x_{2}\left(1-x_{1}-x_{2}\right)\right) .
$$

We have $Q\left(\triangle_{0}\right)=\triangle_{0}$ and

$$
\begin{aligned}
J Q\left(x_{1}, x_{2}\right) & =\operatorname{det}\left[\begin{array}{cl}
2\left(x_{1}+x_{2}\right) & 2 x_{1} \\
-2 x_{2} & 2\left(1-x_{1}-x_{2}\right)
\end{array}\right] \\
& =4\left(x_{1}+x_{2}\right)\left(1-\left(x_{1}+x_{2}\right)\right)+x_{1} x_{2} .
\end{aligned}
$$


The critical set of $Q$ is the ellipse

$$
C=\left\{\left(x_{1}, x_{2}\right): \frac{3}{4}\left(\left(x_{1}+x_{2}\right)-\frac{2}{3}\right)^{2}+\frac{1}{4}\left(x_{1}-x_{2}\right)^{2}=\frac{1}{3}\right\} .
$$

All vertices of $\triangle_{0}$ lie on this ellipse. In complex coordinates

$$
\begin{aligned}
Q(z) & =-\frac{1+i}{4}\left(z^{2}+2 i|z|^{2}-3 \bar{z}^{3}-2(1-i)(z-\bar{z})\right) \\
& =-\frac{1+i}{4}((z+(\sqrt{2}+i) \bar{z})(z-(\sqrt{2}-i) \bar{z})-2(1-i)(z-\bar{z})) .
\end{aligned}
$$

Hence $\bar{Q}$ is properly extendable.

Moreover since the critical set of $Q$ is an ellipse, $Q$ is affinely conjugate to a harmonic quadratic polynomial map. Hence we have obtained an example of a harmonic quadratic polynomial map with an invariant triangle.

ExAmple 2 (the Lotka-Volterra map; see [Św], [BGLL], [GL]). The Lotka-Volterra map is

$$
Q^{0}\left(x_{1}, x_{2}\right)=\left(x_{1}\left(4-x_{1}-x_{2}\right), x_{1} x_{2}\right) .
$$

Let $\triangle=\left\{\left(x_{1}, x_{2}\right): x_{1} \geq 0, x_{2} \geq 0, x_{1}+x_{2} \leq 4\right\}$. We have $Q^{0}(\triangle) \subset \triangle$. Let $h\left(x_{1}, x_{2}\right)=\left(4 x_{1}, 4 x_{2}\right)$. Put

$$
Q\left(x_{1}, x_{2}\right)=h^{-1} \circ Q^{0} \circ h\left(x_{1}, x_{2}\right)=\left(4 x_{1}\left(1-x_{1}-x_{2}\right), 4 x_{1} x_{2}\right) .
$$

Then $Q\left(\triangle_{0}\right) \subset \triangle_{0}$.

The mapping $Q$ is conjugate to the simplex map

$$
F\left(x_{1}, x_{2}, x_{3}\right)=\left(4 x_{1} x_{3}, 4 x_{1} x_{2},\left(x_{1}-x_{2}-x_{3}\right)^{2}\right) .
$$

It is clear that $Q$ cannot be extendable since $Q=0$ on the line $\left\{\left(x_{1}, x_{2}\right)=x_{1}\right.$ $=0\}$. The critical set of $Q$ is the union of two parallel lines $\left\{\left(x_{1}, x_{2}\right): x_{1}=0\right\}$ $\cup\left\{\left(x_{1}, x_{2}\right): x_{1}=1 / 2\right\}$.

EXAMPLE 3 (holomorphic quadratic polynomial maps). The mapping $Q_{0}(z)=z^{2}$ maps the closed triangle $\Delta^{0}$ with vertices $1,(-1+\sqrt{3} i) / 2$, $(-1-\sqrt{3} i) / 2$ into itself.

The proof of this fact is very elementary:

We have

$$
\triangle^{0}=\left\{z=x+i y:-\frac{1}{2} \leq x \leq 1,|y| \leq \frac{1-x}{\sqrt{3}}\right\} .
$$

Hence $z^{2} \in \triangle^{0}$ iff $-\frac{1}{2} \leq x^{2}-y^{2} \leq 1$ and $|2 x y| \leq\left(1-\left(x^{2}-y^{2}\right)\right) / \sqrt{3}$. If $z \in \triangle^{0}$ then

$$
x^{2}-y^{2} \geq x^{2}-\frac{(1-x)^{2}}{3}=\frac{2}{3} x^{2}+\frac{2}{3} x-\frac{1}{3} \geq-\frac{1}{2} .
$$


We have the following equivalence:

$$
2|x||y| \leq \frac{1-|x|^{2}+|y|^{2}}{\sqrt{3}} \Leftrightarrow|y|^{2}-2 \sqrt{3}|x||y|+1-|x|^{2} \geq 0 .
$$

Taking $|x|$ as a parameter we see that the discriminant $\delta$ is equal to

$$
12|x|^{2}-4\left(1-|x|^{2}\right)=16|x|^{2}-4 .
$$

If $|x| \leq \frac{1}{2}$ then $\delta \leq 0$ and so the last displayed inequality holds. If $\frac{1}{2} \leq x \leq 1$ then our inequality is true for

$$
|y| \leq \frac{2 \sqrt{3} x-2 \sqrt{4 x^{2}-1}}{2}=\sqrt{3} x-\sqrt{4 x^{2}-1} .
$$

This is true for $z=x+i y \in \triangle^{0}$ because

$$
\frac{1-x}{\sqrt{3}} \leq \sqrt{3} x-\sqrt{4 x^{2}-1} \text { for } 1 / 2 \leq x \leq 1 .
$$

The dynamics of $Q^{0}$ on $\triangle^{0}$ under iteration is the following:

All points of $\triangle^{0}$ except the vertices are attracted to 0 , the vertex 1 is a repelling fixed point and the vertices $(-1+\sqrt{3} i) / 2$ and $(-1-\sqrt{3} i) / 2$ form a repelling cycle of period 2 .

Let $Q_{\lambda}(z)=z^{2}+\lambda z, \lambda \in \mathbb{C}$. If $|\lambda|<1 / 2$ then for sufficiently small $\varepsilon>0$, $Q_{\lambda}$ maps the disc $B(0, \varepsilon)$ into $B(0, \varepsilon / 2)$. Hence it maps $\varepsilon \triangle^{0}$ into itself. In this case the whole triangle $\varepsilon \triangle^{0}$ is attracted to 0 under iterations of $Q_{\lambda}^{0}$. Hence, for $\lambda=0$ we have two different invariant triangles $\triangle^{0}$ and $\varepsilon \triangle^{0}$ with different dynamics.

The simplex maps corresponding to $\left.Q_{0}\right|_{\triangle^{0}}$ and $\left.Q_{0}\right|_{\varepsilon \triangle^{0}}$ cannot be conjugate.

There are many holomorphic quadratic polynomial which do not have invariant triangles. A necessary condition for $Q$ to have such a triangle is that $Q$ must have a bounded invariant component of the Fatou set.

\section{References}

[BGLL] F. Balibrea, J. L. García Guirao, M. Lampart and J. Llibro, Dynamics of a Lotka-Volterra map, Fund. Math. 191 (2006), 265-279.

[CG] L. Carleson and T. W. Gamelin, Complex Dynamics, Springer, New York, 1993.

[CHRS] W. D. Crowe, R. Hasson, P. J. Rippon and P. E. D. Strain-Clark, On the structure of the Mandelbar set, Nonlinearity 2 (1989), 541-553.

[GL] J. L. García Guirao and M. Lampart, Transitivity of the Lotka-Volterra map, Discrete Contin. Dynam. Systems Ser. B 9 (2008), 75-82.

[Li1] E. Ligocka, On the complexification and iteration of quasiregular polynomials which have algebraic degree two, Fund. Math. 186 (2005), 269-285.

[Li2] - On the complexification of real-analytic polynomial mappings of $\mathbb{R}^{2}$, Ann. Polon. Math. 88 (2006), 119-125. 
[Li3] E. Ligocka, On the dynamics of extendable polynomial endomorphisms of $\mathbb{R}^{2}$, Ann. Polon. Math. 90 (2007), 37-50.

[Ly] Yu. I. Lyubich, Mathematical Structures in Population Genetics, Springer, 1992.

[MSU] M. T. Menzel, P. R. Stein and S. M. Ulam, Quadratic transformations I, Rep. LA 2305, Los Alamos, 1955.

[N] S. Nakane, Connectedness of the tricorn, Ergodic Theory Dynam. Systems 13 (1993), 349-356.

[NS] S. Nakane and D. Schleicher, On multicorns and unicorns. I. Antiholomorphic dynamics, hyperbolic components and real cubic polynomials, Int. J. Bifur. Chaos Appl. Sci. Engrg. 13 (2003), 2825-2844.

[Sha] I. R. Shafarevich, Foundations of Algebraic Geometry, Nauka, Moscow, 1988 (in Russian).

[She] T. Sheil-Small, Complex Polynomials, Cambridge Univ. Press, 2002.

[Św] G. Świrszcz, On a certain map of a triangle, Fund. Math. 155 (1998), 45-57.

[U] K. Uchimura, Dynamics of symmetric polynomial endomorphisms of $\mathbb{C}^{2}$, Michigan Math. J. 55 (2007), 483-511.

Pasteura 4/6 m. 31

02-093 Warszawa, Poland

E-mail: elig@mimuw.edu.pl

Received 6.1.2009

and in final form 27.1.2009 